\title{
Cualidades del personal de enfermería en salud mental para las consejerías en adicción a drogas 1
}

Daniel Martínez Esquivel ${ }^{2}$

Institución: Universidad de Costa Rica

\author{
ENSAYO \\ RESUMEN
}

El consumo de sustancias es un problema de salud pública que debe ser atendido de manera interdisciplinaria a partir de diferentes estrategias de acción como la consejería en adicción a drogas. La incursión de la enfermería en salud mental fortalece los dispositivos de tratamiento a partir de su ser, saber y hacer. El presente ensayo responde a ¿cuáles son las cualidades del personal de enfermería en salud mental para las consejerías en adicción a drogas? La enfermería en salud mental es una profesión autónoma que se define por fundamentos teóricos propios de la disciplina, los cuales le otorgan conocimientos y competencias características que le permiten realizar aportes clave en la formación en consejería a partir de las habilidades y destrezas que manifiesta. Se concluye que los componentes teóricos que forman el cuerpo de conocimientos, le permiten a la enfermería en salud mental desarrollar una personalidad capaz de entender la adicción y sus necesidades para brindar una atención de calidad a través de las consejerías.

Palabras clave: atención-en-salud-mental; dependencia-a-drogas; enfermería.

${ }^{2}$ Enfermero en Salud Mental. Escuela de Enfermería, Universidad de Costa Rica. Costa Rica. Correo electrónico: dtinez@gmail.com 


\title{
Qualities of the mental health nursing staff for drug addiction counseling ${ }^{1}$
}

Daniel Martínez Esquivel ${ }^{2}$

Institution: University of Costa Rica

\author{
ESSAY
}

ABSTRACT

The substance used is a public health problem must be addressed in an interdisciplinary way, based on different actions such as drugs addiction counseling. The incursion of the nursing in mental health strengthens the treatment devices based on their being, knowing and doing. This essay aims to answer, what are the qualities of nursing in mental health for drugs addiction counseling? Mental health nursing is an autonomous profession that is defined by the theoretical foundations of the discipline, which provide knowledge and skills that allow you to make key contributions in counseling training based on your own abilities. Conclusion. The theoretical components that make up the body of knowledge allow mental health nursing to develop a personality capable of understanding the addiction and it needs to provide quality care through counseling.

Keywords: addiction; counseling; nursing.

DOI 10.15517/revenf.v0iNo. 37.34726

${ }^{1}$ Date of receipt: October 1, 2018

Date of acceptance: November 10, 2018

${ }^{2}$ Nurse in Mental Health. School of Nursing, University of Costa Rica. Costa Rica. E-mail: dtinez@gmail.com 


\section{Qualidades da equipe de enfermagem em saúde mental para aconselhamento sobre dependência de drogas ${ }^{1}$}

Daniel Martínez Esquivel ${ }^{2}$

Instituição: Universidade de Costa Rica

\section{ENSAIO}

RESUMO

O uso de substâncias é um problema de saúde pública que deve ser abordado de forma interdisciplinar com base em diferentes estratégias de ação, como o aconselhamento Aconselhamento em toxicodependência. A incursão da enfermagem em saúde mental fortalece os equipes de tratamento baseados em seu ser, saber e fazer. O presente ensaio pretende responder quais são as qualidades do pessoal de enfermagem em saúde mental para o aconselhamento em toxicodependência? Enfermagem em saúde mental é uma profissão autônoma que é definida pelos fundamentos teóricos da disciplina, lhe dão conhecimento e competições que lhe permitem fazer contribuições essenciais no treinamento de aconselhamento com base nas habilidades que ele manifesta. Conclusão. Os componentes teóricos du corpo de conhecimento da enfermagem em saúde mental permiten-le desenvolva uma personalidade capaz de compreender da toxicodependência e suas necessidades de prestar um atendimento de qualidade por meio do aconselhamento.

Palavras-chave: aconselhamento; dependencia; enfermagem.

DOI 10.15517/revenf.v0iNo. 37.34726

${ }^{1}$ Data de recebimento: $1^{\circ}$ de outubro de 2018.

Data de aceitação: 10 de novembro de 2018

${ }^{2}$ Enfermeiro em Saúde Mental. Escola de Enfermagem, Universidade de Costa Rica. Costa Rica. Correio eletrônico: dtinez@gmail.com 


\section{INTRODUCCIÓN}

Este ensayo es producto de la autorreflexión en el curso de "Formación en consejería en alcohol y otras drogas", el cual forma parte del Programa de capacitación y certificación del recurso humano en tratamiento y atención en drogas - Costa Rica (PROCCERTRAD-CR), y en el que participaron personas profesionales y no profesionales (esta indicación responde no al interés de discriminar a alguien o establecer una línea de status social, sino al simple hecho de caracterizar la población que se reúne con un mismo objetivo: ser consejeros.

La idea es que la tarea de consejería en adicción a drogas prolifere en los centros de tratamiento de adicciones a nivel nacional, de manera que responda a los alarmantes datos epidemiológicos existentes, lo cual vuelve imprescindible la intervención desde diferentes paradigmas para atender las necesidades específicas de la población.

En el caso de Costa Rica, de acuerdo con la IV Encuesta Nacional sobre Consumo de Drogas en Población de Educación Secundaria ${ }^{1}$, se determinó que el alcohol es la sustancia más consumida entre la población adolescente, seguida de la marihuana, y el tabaco, en tercer lugar, a lo que se suma que -según el Informe de Situación Nacional sobre Drogas y Actividades Conexas ${ }^{2}$ en seis años las atenciones brindadas por el Instituto sobre Alcoholismo y Farmacodepencia (IAFA) aumentaron un 73,6\%: a pesar de que aducen este incremento se debe a la ampliación en la cobertura de los servicios que ofrece, no se puede negar que el abordaje de las adicciones tiene carácter de emergencia.

Las estadísticas anteriores evidencian cómo las drogas se han convertido en un determinante de la salud que se posiciona como una respuesta al resquebrajamiento social que provoca inequidades en salud. Al respecto, Wilkinson y Marmot ${ }^{3}$ mencionan que el uso y abuso de las drogas está asociado con marcadores de desventaja social y económica donde los usuarios buscan una salida ilusoria para afrontar el dolor, la adversidad y el estrés. Por lo tanto, las acciones en función de la promoción de la salud son necesarias para aliviar el sufrimiento psíquico.

En torno a la adicción, comprenderla no es sencillo, por ende, es importante clarificar algunos conceptos básicos relacionados con el tema de consumo de drogas. Primeramente, el uso de sustancias psicoactivas se define como un consumo ocasional o frecuente que se podría conocer como consumo social ${ }^{4}$, mientras que el abuso es utilizar una sustancia psicoactiva por iniciativa propia de una manera que se desvía de los patrones sociales aceptados, y la dependencia es el uso repetido y fuera de control en el que puede existir o no sometimiento físico ${ }^{5}$.

En cuanto al concepto de adicción, converge con el de dependencia; sin embargo, en el Manual diagnóstico y estadístico de los trastornos mentales DSM-5, ${ }^{6}$ es sustituido por trastorno de consumo de sustancias, como una forma de describir de manera dinámica esta enfermedad; por lo tanto, al hacer referencia al uso, abuso y dependencia de drogas se describe un problema de salud pública que tiene repercusiones individuales, familiares, comunales, sociales, políticas y económicas, al punto de que se ha convertido en una pandemia descontrolada que ha involucrado a millones de personas que entregaron sus vidas a las sustancias psicoactivas a causa de esta enfermedad ${ }^{6}$. 
Ante tal panorama, la intervención del consejero se centra en enfrentar la adicción por medio del proceso de recuperación y a través de la abstinencia. Cualquier persona que sea entrenada puede convertirse en consejero; sin embargo, se considera, que el ser, saber y hacer de la enfermería en salud mental permitiría realizar aportes importantes a la consejería en alcohol y otras drogas desde sus competencias; por ello, considerando lo mencionado, en el presente ensayo se pretende responder al siguiente problema, ¿cuáles son las cualidades del personal de enfermería en salud mental para las consejerías en adicción a drogas?

\section{DESARROLLO}

La salud mental debe ser considerada desde una perspectiva social, cuya base sea el bienestar y el funcionamiento efectivo de las personas en sociedad $^{7}$, sin dejar de lado que es un derecho innegable, vinculado con conceptos como ciudadanía, libertad, democracia y solidaridad, de modo que se garantice el desarrollo personal y social ${ }^{8}$, máxime considerando que la salud mental es de y para todas las personas y su funcionamiento es clave para la autorrealización y la calidad de vida relacionada a la salud.

En torno a la enfermería en salud mental y psiquiatría se define como "un área especializada de la práctica de Enfermería que emplea una variedad de teorías explicativas del comportamiento humano como su ciencia y el uso apropiado de la misma como su arte" 9 . Stuart y Laraia ${ }^{4}$ se refieren a ella como el proceso interpersonal que promueve y mantiene el comportamiento del paciente visualizado como una persona, familia o comunidad.

Al respecto, Neuman y Fawcett ${ }^{10}$, la consideran la única profesión de la salud capaz de atender todas las variables que afectan a las personas en su entorno, cuyo objetivo es valorar a la persona en relación con el ambiente para mantener el nivel más óptimo de salud del individuo, la familia y la comunidad a partir de la promoción y la prevención. Por último, para Malvárez ${ }^{11}$ es una ciencia social que tiene como objetivo el cuidado humano a partir de las vivencias de la salud, no para curar sino para confortar el impacto de la enfermedad y potenciar sus capacidades debilitadas.

Las definiciones expuestas dotan a dicha especialidad de un carácter social en el entendido de que busca estudiar los fenómenos para abordar las situaciones de salud. Conocer, analizar e interpretar las necesidades permite comprender a las personas y a la sociedad visualizando su función social y sensibilidad para estudiar el fenómeno de las drogas.

Todo lo anterior se apoya en una serie de características que debe desarrollar aquella persona que se prepare para ejercer la enfermería en salud mental fundamentadas en el uso de sí misma, las cuales son esenciales en el proceso de cuidado y dotan a la disciplina de un carácter terapéutico; entre tales características personales se identifica las siguientes: conciencia de sí, claridad de valores, exploración de sentimientos, servir como modelo de conducta, altruismo, ética y responsabilidad ${ }^{4}$, las cuales son descritas a continuación respecto de su vínculo con la consejería en alcohol y otras drogas.

En cuanto a la conciencia de sí, Eckroth-Bucher ${ }^{12}$ refiere que le permite al enfermero(a) en salud mental una mejor comprensión y aceptación de su Yo, lo cual le facilita posicionarse frente a la adicción y al reconocimiento de las diferencias y singularidades de las personas que se permite atender, mientras que por medio de la 
autenticidad $^{4}$, se abre a la exploración de sus propias experiencias relacionadas con el uso, consumo o abuso de drogas para construir un criterio respecto de la problemática y la forma en que puede ejecutar sus acciones de cuidado.

Lo anterior pretende responder a la pregunta ¿cuál es el sistema de valores que dirige mi vida? Los valores son conceptos con una connotación de bienestar que se forman a partir de las experiencias de vida y sus enseñanzas: su claridad fortalece la honestidad de la práctica clínica de la enfermería en salud mental y favorece la seguridad de la persona usuaria ${ }^{4}$. A lo anterior debe agregarse que los profesionales ejercen su profesión a partir de un código de ética que se rige por los derechos humanos, la equidad de género, la libertad y la democracia que permite crear un entorno personificado para cada caso que atiende.

El sistema de valores se construye a partir de la formación académica pero también de manera individual a partir de la exploración de sentimientos, por ello es que la enfermería en salud mental se debe presentar de manera abierta para entender las necesidades de salud, con la capacidad de experimentar los sentimientos de los demás para reconocer los propios ${ }^{4}$. El saber reconocer los propios sentimientos ante un tema en específico provoca acciones eficientes y eficaces de (auto)cuidado.

Retomando el primer aspecto, cuanto mayor sea el conocimiento de sí, mayor será la de los profesionales para cuidar su salud y ser objeto de mediación del cuidado, hasta convertirse en un modelo de conducta. De acuerdo con Stuart y Laraia ${ }^{4}$, la influencia que se tiene sobre los(as) usuarios(as) es intenso, lo cual dota a la enfermería en salud mental de un deber innato de reflejar comportamientos adaptativos para que su credibilidad no sea cuestionada. Durante la intervención, la relación es simbiótica donde ambas partes crecen de manera conjunta.

Cuando los profesionales se preocupan por satisfacer sus necesidades, desarrollan la capacidad de preocuparse por el bienestar de otras personas: tal característica se conoce como altruismo ${ }^{4}$. En la enfermería en salud mental, ser altruista le permite a los profesionales escuchar y entender cuáles son las necesidades que demanda la persona para satisfacerlas de la manera más acertada por medio de las acciones de cuidado.

Cada acción se fundamenta en la ética que conlleva la práctica profesional: en este caso, está en función de sus virtudes, muy ligada a la emisión de juicios y toma de decisiones, enfatiza en los conocimientos, la sensibilidad y la responsabilidad de tener la capacidad de identificar las situaciones de salud y responder de manera efectiva con los cuidados específicos que requieren las personas ${ }^{13}$.

Las características mencionadas forman una personalidad de la enfermería en salud mental que se manifiestan en su quehacer y que se ajustan de una manera adecuada a las competencias de la práctica de consejería en adicción a drogas, partiendo del hecho de que los profesionales en adicción trabajan desde distintas disciplinas pero compartiendo un entendimiento del proceso adictivo ${ }^{14}$.

Además, de manera autónoma, la comprensión de este fenómeno social en el cotidiano de cada persona parte de un fundamento sistémico: King ${ }^{16}$ propone el supuesto de que las personas son seres sociales que interaccionan de manera dinámica en tres sistemas -personal, interpersonal y social- considerados abiertos, ya que constantemente generan intercambios que definen el universo de las personas. Sin embargo, enfatiza que el sistema interpersonal es clave para el hacer de la enfermería en salud mental en cuanto permite la interacción de dos personas que 
organizan las acciones de cuidado a partir del establecimiento de metas comunes ${ }^{16}$ :al igual que en la práctica profesional de la consejería, la interacción con el usuario se vuelve una herramienta necesaria para examinar las opciones de tratamiento, priorizar las necesidades y formular las metas acordadas ${ }^{14}$.

Respecto del usuario, Neuman et al. ${ }^{10}$ lo visualizan como un sistema compuesto por variables fisiológicas, psicológicas, socioculturales, de desarrollo y espirituales que se manifiestan en la estructura básica, protegida por la línea flexible de defensa, la línea normal de defensa y las líneas de resistencia de todos los estresores presentes en el entorno: una dinámica adaptativa de esta estructura garantiza un mejor nivel de salud en las personas.

Las líneas de defensa, flexible y normal, actúan como un sistema de amortiguación para la persona, es decir, previenen la invasión de estresores al sistema cliente usuario al que le brindan protección. El impacto de múltiples estresores tiene el potencial de reducir su efectividad haciendo que el sistema cliente presente síntomas, inestabilidad o enfermedad. Por último, las líneas de resistencia son activadas en el momento en que la línea normal de defensa es invadida por los estresores del ambiente, cuyo propósito es proteger la estructura básica ${ }^{10}$.

En la adicción, el sistema cliente se ve afectado por estresores como el aburrimiento, la culpa y la vergüenza, los disparadores externos e internos, la ansiedad, la ira, la ambivalencia, el estrés, entre otros ${ }^{17}$, que Neuman et al. ${ }^{10}$ han identificado como intrapersonales, interpersonales y extrapersonales, los cuales provocan daños en las líneas de defensa que afectan directamente la estructura básica de la persona, dificulta su proceso de recuperación y fomenta la recaída.

Para fortalecer las líneas de defensa, los profesionales pueden emplear recursos de la consejería en adicción a drogas apoyándose en la prevención que no solo identifica las causas sino que indica las acciones requeridas en cada caso.

En torno a lo mencionado, hay tres niveles de prevención que pueden ser utilizados de forma simultánea según las condiciones de la persona ${ }^{10}$ :

1.Prevención primaria: su propósito es proteger la línea normal de defensa de la persona a partir de la promoción de la salud por medio de la prevención del estrés y la disminución de los factores de riesgo. La prevención primaria se utiliza cuando se conoce el riesgo, pero no se presenta ninguna reacción o síntoma, y se enfoca en reducir los factores de riesgo para disminuir una posible reacción.

2.Prevención secundaria: protege la estructura básica al fortalecer las líneas de resistencia. Su propósito es brindar tratamiento apropiado sobre los síntomas para conservar la estabilidad del sistema y la conservación de la energía. Se debe aprovechar todos los recursos del sistema, internos y externos, para reducir la reacción a los estresores.

3.Prevención terciaria: protege la recuperación del sistema cliente a su mejor estado de salud posible. Su propósito es mantener un nivel óptimo de salud por medio de las fortalezas y la conservación de la energía de la persona. 
La capacidad de dimensionar a cada persona como un sistema dinámico permite a la enfermería en salud mental acercarse al concepto de multicausalidad de la adicción. Al seguir diferentes líneas etiológicas, comprende este fenómeno tan complejo e individual de manera que atiende cada una de sus necesidades específicas.

Gordon citada por Fornés-Vives ${ }^{18}$ plantea una clasificación centrada en los patrones de salud que brindan un entendimiento de las áreas que podrían afectarse a causa de la adicción y/o fortalecerse en el proceso de recuperación. Los patrones funcionales de salud "son aquella respuesta que describe el grado de función corporal que contribuye a mantener el estado de salud, la calidad de vida y la realización del potencial humano"; se dividen en afrontamiento-tolerancia al estrés, autopercepción-autoconcepto, cognitivo-perceptivo, valores-creencias, actividad-ejercicio, eliminación, nutricional-metabólico, percepción-mantenimiento de la salud, rol-relaciones, sexualidad-reproducción y sueño-descanso ${ }^{18,19}$.

Desde las consejerías, la enfermería en salud mental es más receptiva a identificar situaciones que afectan el estado general de salud de la persona, lo que facilita las funciones de coordinación de servicio y de manejo de caso $^{14}$, cuyo propósito será atender los patrones de salud que sufren alguna alteración intimamente ligada al consumo de sustancias y referir a diferentes profesionales la atención de las otras demandas.

La puesta en práctica en las consejerías en adicción a drogas de los fundamentos teóricos que se han mencionado en el presente artículo, está ligada a las funciones que desempeña la enfermería en salud mental en el ejercicio profesional, definidas por Peplau ${ }^{20}$ como roles, los cuales son asignados a los profesionales del área como método de acción para afrontar las necesidades del cliente. En total, se identifica seis roles específicos que fortalecen el hacer disciplinar en el tratamiento del trastorno por consumo de sustancias: rol de extraño, de persona-recurso, de enseñanza, de las funciones de liderazgo, sustitutivos y de asesor.

En cuanto al de extraño, se ejerce en el primer contacto con la persona que solicita el servicio de atención; en las consejerías, es clave durante la evaluación clínica debido a que es el momento de acercamiento sistémico en el que se utiliza destrezas como el respeto, la empatía y la autenticidad ${ }^{14}$ : cuando los profesionales son extraños a los usuarios parte del principio de aceptarlo tal cual consiste en entenderlo como alguien emocionalmente apto para relacionarse y conocerlo ${ }^{20}$.

Cuando la relación con el cliente se establece, los profesionales en salud mental se convierten en personas-recurso que ofrecen un servicio que merece ser explotado ${ }^{20}$. En este sentido, proporcionan respuestas a las necesidades descubiertas, plasmadas en el plan de tratamiento formulado en función de las metas y objetivos que hayan acordado mutuamente ${ }^{14}$.

La implementación del plan de tratamiento tiene como componente relevante los procesos educativos al cliente, la familia y la comunidad ${ }^{14}$. Los roles de enseñanza se desarrollan a partir del interés en torno a un tema para crear una respuesta útil en los procesos de prevención y recuperación que mejoren las experiencias individuales y grupales $^{20}$. Para lograr tal impacto positivo, los profesionales en salud mental desempeñan el rol de las funciones de liderazgo, el cual no depende de la autoproclamación personal, sino que es asignado por los clientes en su necesidad de recibir orientación para sus dificultades ${ }^{20}$. 
En relación con los tipos de liderazgos, Peplau ${ }^{20}$ menciona tres -democrático, autocrático y liberal-: de acuerdo con las consejerías, ninguno es mejor que otro, sino que su manifestación dependerá de la etapa de tratamiento de la persona.

Junto al liderazgo, el rol de asesor tiene que ver con la forma en que se responde a las demandas de la persona, incluso está relacionado con la ayuda que recibe para que recuerde plenamente lo que le sucede al integrarlo en su vida para que tome las medidas correctivas que mejoren su salud. ${ }^{20}$ En la consejería, la asesoría -basada en la Filosofía de los 12 pasos- se dirige a comprender la adicción como una enfermedad que genera impotencia y afecta todas las áreas de la vida ${ }^{21}$.

Por último, los roles sustitutivos se presentan cuando la persona -de manera inconsciente- considera a los profesionales en salud mental como personas distintas, por lo que el profesional debe ayudar al usuario a cobrar consciencia sobre quién es la persona que presta el servicio, sin que acepte las falsas características que se le otorgan $^{20}$, ya que evitar los roles sustitutivos converge con las responsabilidades profesionales y éticas del consejero que demandan conductas adecuadas ${ }^{14}$.

Cada una de las cualidades de la enfermería en salud mental que se mencionaron en este artículo son exitosas a partir del desarrollo de la relación enfermera(o)-cliente, la cual es dinámica, flexible y terapéutica: según Peplau $^{19,20}$, se caracteriza por la interacción de cuatro fases -orientación, identificación, explotación y resolucióncentradas en las necesidades de la persona. Dado lo anterior, los profesionales tienen la obligación de desarrollar las habilidades y destrezas adecuadas para ejercer la profesión fundamentada en su enfoque teórico; por ende, las consejerías en adicción a drogas deben nutrirse de conocimientos interdisciplinarios que resalten el valor de todas aquellas disciplinas que tienen la intención de intervenir en este problema de salud pública.

\section{CONCLUSIONES}

Las personas que tienen un trastorno por consumo de sustancias deben tener acceso a diversos servicios que garanticen su bienestar. Cada servicio debe componerse por diferentes profesionales que hagan un aporte desde su especialidad en la práctica clínica, basados en el trabajo de equipo.

La enfermería en salud mental se posiciona como una disciplina respaldada por un marco teórico que justifica su ser, saber y hacer, de modo que sea competente para ofrecer acciones de cuidado a quienes sufren producto de la adicción, fundamento que le permite disfrutar de diferentes características que se ajustan al perfil de consejería en adicción a drogas.

La consejería en adicción a drogas es un área de especialización útil que no debe ser desestimada o sustituida en ningún momento; por el contrario, debe ofrecerse como una opción para las personas en respuesta a sus necesidades.

Declaración conflicto de intereses: el autor declara que no tiene ningún conflicto de intereses de tipo personal, económico, ni de ninguna otra índole. 


\section{REFERENCIAS BIBLIOGRÁFICAS}

1. IAFA. IV Encuesta Nacional sobre consumo de drogas en la población de educación secundaria. Costa Rica: IAFA; $2015 . \quad$ Disponible en: https://www.iafa.go.cr/images/descargables/conocimiento/Consumo $\% 20 \mathrm{de} \% 20 \mathrm{drogas} \% 20 \mathrm{en} \% 201 \mathrm{a} \% 20 \mathrm{juventud}$ \%20escolarizada\%202015\%20Costa\%20Rica,\%202017.pdf

2. ICD. Informe de Situación Nacional sobre Drogas y Actividades Conexas. Costa Rica: ICD; 2016. Disponible en:

https://www.icd.go.cr/portalicd/images/docs/uid/informes/SituacionNac/CR_SituacionNacional_DrogasActivCon exas_2017.pdf

3. Wilkinson R, Marmot M. Determinantes sociales de la salud: los hechos irrefutables. Ginebra: OMS; 2003. Disponible en: http://www.bvsde.paho.org/bvsacd/cd90/1008WILdet.pdf

4. Stuart G, Laraia M. Enfermería psiquiátrica. Principios y práctica. Octava Edición. Madrid: Elsevier; 2006.

5. Sadock B, Sadock V. Kaplan \& Sadock. Manual de bolsillo de psiquiatría clínica. Quinta Edición. Barcelona: Wolters Kluwer Health; 2011.

6. American Psychiatric Association. Guía de consulta de los criterios diagnósticos del DSM-5. Washington, DC: APA; 2014.

7. OMS. Promoción de la Salud Mental: Conceptos, evidencia emergente, práctica. Ginebra: OMS; 2004. Disponible en: https://www.who.int/mental health/evidence/promocion de la salud mental.pdf

8. Galli V. Salud Mental, definiciones y problemas. Buenos Aires: Documento de trabajo de la Dirección Nacional de Salud Mental del Ministerio de Salud.

9. American Nurses Association. Scope and standards of psychiatric-mental health clinical nursing practice. Washington D.C.: The Association; 2000. DOI: 10.3912/OJIN.Vol20No01Man01

10. Neuman M, Fawcett. The Neuman Systems Model. Fourth Edition. New Jersey: Prentice Hall; 2002.

11. Malvárez S. El reto de cuidar en un mundo globalizado. Texto \& Contexto Enfermagem. 2007; 16 (003): 520530. DOI: http://dx.doi.org/10.1590/S0104-07072007000300019

12. Eckroth-Bucher M. Philosophical basis and practice of self-awareness in psychiatric nursing. J Psychiatr Nurs Assoc. 1998; 4:105. 
13. Fry S, Johnstone M. Ética en la práctica de enfermería. Una guía para la toma de decisiones éticas. México: Manual Moderno. 2010.

14. Centro para el Tratamiento de Abuso de Sustancias. Conocimientos, Destrezas, y Actitudes de la Práctica Profesional. Serie de Publicaciones para Asistencia Técnica (TAP) 21. Rockville: DHHS; 2010. Disponible en: https://store.samhsa.gov/system/files/sma12-4171.pdf

15. Orem D. Normas prácticas en Enfermería. Madrid: Ediciones Pirámide S.A.;1983.

16. King I. Enfermería como profesión. Filosofía, principios y objetivos. México: Editorial Limusa S.A.; 1984.

17. Zarza MJ, Martí J, Botella A, Vidal A, Ribeiro B, Bisetto D. Modelo Matrix: tratamiento ambulatorio intensivo del consumo de sustancias estimulantes. Manual del usuario.; 2013. Disponible en: http://www.matrix.edrogas.es/ServletDocument;jsessionid=967F4958020F3156B809F5FED5DD35F6?document $=226$

18. Fornés-Vives J, Carballal-Balsa MC. Enfermería de Salud Mental y Psiquiatría. Guía práctica de valoración y estrategias de intervención. Primera Edición. Madrid: Editorial Médica Panamericana; 2001.

19. Peplau H. Relaciones interpersonales en Enfermería. Un marco de referencia conceptual para la enfermería psicodinámica. Barcelona: Salvat Editores S.A.; 1990.

20. Herrera-Arce A, Betolaza-López E, Murúa-Navarro F, Martínez H, Jiménez-Lerma JM. Enfermería en adicciones: El modelo teórico de H. Peplau a través de los patrones funcionales de M. Gordon. A propósito de un caso práctico.Trastornos adictivos. 2003; 5 (2): 58-74. DOI: https://doi.org/10.1016/S1575-0973(03)78556-0

21. Alcoholics Anonymous World Services. Los Doce Pasos de Alcohólicos Anónimos. Material de Servicio de la Oficina de Servicios Generales. Alcoholics Anonymous Publishing, Inc.; 1981. Disponible en: https://www.aa.org/assets/es_ES/smf-121_sp.pdf 